\title{
Classification of Hazelnut Kernels by Using Impact Acoustic Time-Frequency Patterns
}

\author{
Habil Kalkan, ${ }^{1}$ Nuri Firat Ince, ${ }^{2}$ Ahmed H. Tewfik, ${ }^{2}$ Yasemin Yardimci, ${ }^{1}$ and Tom Pearson ${ }^{3}$ \\ ${ }^{1}$ Informatics Institute, Middle East Technical University, 06531 Ankara, Turkey \\ ${ }^{2}$ Department of Electrical and Computer Engineering, University of Minnesota, MN 55455, USA \\ ${ }^{3}$ Agricultural Research Service, United States Department of Agriculture, KS 66502, USA \\ Correspondence should be addressed to Yasemin Yardimci, yardimy@ii.metu.edu.tr
}

Received 17 January 2007; Revised 7 July 2007; Accepted 8 October 2007

Recommended by Hugo Van hamme

\begin{abstract}
Hazelnuts with damaged or cracked shells are more prone to infection with aflatoxin producing molds (Aspergillus flavus). These molds can cause cancer. In this study, we introduce a new approach that separates damaged/cracked hazelnut kernels from good ones by using time-frequency features obtained from impact acoustic signals. The proposed technique requires no prior knowledge of the relevant time and frequency locations. In an offline step, the algorithm adaptively segments impact signals from a training data set in time using local cosine packet analysis and a Kullback-Leibler criterion to assess the discrimination power of different segmentations. In each resulting time segment, the signal is further decomposed into subbands using an undecimated wavelet transform. The most discriminative subbands are selected according to the Euclidean distance between the cumulative probability distributions of the corresponding subband coefficients. The most discriminative subbands are fed into a linear discriminant analysis classifier. In the online classification step, the algorithm simply computes the learned features from the observed signal and feeds them to the linear discriminant analysis (LDA) classifier. The algorithm achieved a throughput rate of 45 nuts/s and a classification accuracy of $96 \%$ with the 30 most discriminative features, a higher rate than those provided with prior methods.
\end{abstract}

Copyright (C) 2008 Habil Kalkan et al. This is an open access article distributed under the Creative Commons Attribution License, which permits unrestricted use, distribution, and reproduction in any medium, provided the original work is properly cited.

\section{INTRODUCTION}

Tree nuts are extensively used in the food industry. Environmental conditions and processing procedures may decrease nut quality by causing cracks or damage to the shell. Damage to the shell of the nut kernel increases the likelihood that fungi will infect the kernels. Fungal infestation can cause aflatoxin formation, which is a type of mycotoxin that is linked to various health problems including liver cancer [1]. Therefore, nuts with shell damage should be separated from nuts with regular shells. This same problem affects many different types of tree nuts such as almonds, pecans, hazelnuts, pistachio nuts, and so on. Initial attempts at separation of fungal damaged food items from undamaged ones go back to the studies of Pearson [2]. For pistachio nuts, Pearson showed that nearly all the aflatoxin contaminated pistachios are either caused by bird damage or insects before harvesting or due to early split. Pearson [3] used a machine vision system to classify pistachio nuts into 3 categories such as stained (caused by early splitting), unstained, or moderately stained, with an average classification error of $11 \%$. After removing stained pistachio nuts from unstained ones, the aflatoxin contamination level of pistachio nut is reduced from 4.8-8.6 range to $0.04-2.5 \mathrm{ppb}$ [4].

In another application of tree nut sorting, a high speed sorter based on impact acoustics was developed to separate the pistachio nuts with closed shells from the ones with cracked shells by using the features that were extracted from impact sound signals [5]. This system was improved by using the eigenvalues of mel-cepstrum coefficients and sound amplitudes [6] resulting in a classification accuracy of $97.8 \%$. While this system was primarily designed for separating open and closed shell pistachio nuts, it was shown to provide a feasible method for detecting hazelnuts with cracked shells [7] as well.

Hazelnut quality in the market is mainly measured by the ratio of inner kernel weight to the shell weight. Hence farmers separate the empty hazelnuts from fully developed ones before selling the nuts. A mechanical device working with an air fan is used for this purpose. The air fan deflects 
the hazelnuts with lower weight and the rest of the hazelnuts are accepted as fully developed. This system is unable to determine the nuts with cracked shells because hazelnuts with cracked shell have weights that are very similar to hazelnuts with regular shell. The acoustic sorter system described above is used to separate empty hazelnuts from fully developed nuts in [7] and $97.5 \%$ of these hazelnuts are correctly classified by using 70 features. These features are extracted from the short time variances of signal segments, maximum signal amplitude, spectral peak locations, and the parameters of a Weibull distribution approximation of the envelope of the impact signal parameters. The same features were used for cracked and regular shell hazelnut separation and $94.47 \%$ classification accuracy was obtained. However, this type of algorithm is computationally complex and therefore hard to implement in real time. The results obtained in [7] show the importance of time and frequency features in impact acoustics classification. In order to reduce computational complexity and achieve error rates similar to [7], we recently used an undecimated wavelet transform to classify hazelnuts with regular shell and cracked shell [8]. The most discriminative subbands are manually selected and their energies are used for classification in [8]. A $91.8 \%$ classification rate is achieved with nearly 20 features. Although the computational complexity is reduced with this approach, the classification accuracy is poor compared to [7].

In this study, we propose an adaptive time-frequency ( $t$ $f$ ) analysis approach based on a local discriminant basis algorithm similar to that used in [9-11] to select the most relevant time segments and subbands to maximize classification performance. For this purpose, we combine local cosine packets and wavelet transform which are subsequently used for time and frequency plane feature selection. A schematic diagram summarizing our approach is given in Figure 1. In particular, the local cosine packet analysis is used along the time axis with a pyramidal tree to segment the signals such that the spectral distances in the selected time windows are maximized between classes. A Kullback-Leibler distance was used to estimate the distance between the spectrum of cracked and undamaged hazelnut acoustics. In the next step in each selected time segment, an undecimated wavelet transform is implemented to select the most discriminant subbands. Unlike the algorithm proposed in $[10,11]$ that uses fixed frequency bands, we enhance the frequency axis segmentation by using an undecimated wavelet transform in each adapted time segment. Accordingly, the proposed technique requires no prior knowledge of the relevant time and frequency locations. All these segmentation procedures are executed automatically in an offline manner. As a final step the $t$ - $f$ features are sorted according to a cost function and fed to a linear discriminant. In order to asses the efficiency of different feature selection approaches, we compare two different methods. In particular, the resulting $t$ - $f$ features are sorted by using Fisher discrimination on the pruned tree or processed by the correlation-based feature selection algorithm of [12] implemented on the full tree. The features selected by both algorithms are then fed into the linear discriminant analysis classifier.
The paper is organized as follows. In the next section, the data acquisition system and sample selection procedure are given. The procedures for constructing the time-frequency plane segmentations and the advantages of using undecimated wavelet transform are described in Section 3. Experimental results and conclusions are given in Sections 4 and 5, respectively.

\section{MATERIALS}

\subsection{System description}

The impact acoustic recording system (Figure 2) consists of a pipe, an impact plate, and a microphone. Hazelnut kernels are dropped on an impact plate through the pipe. The impact acoustic signal generated by the system is captured by a microphone and processed by a PC. A stainless steel plate with dimensions $7.5 \times 15 \times 2 \mathrm{~cm}$ is used as the impact plate. The impact plate is fixed to the ground at a $120^{\circ}$ angle. This angle prevents the nuts from making multiple impacts. The microphone is sensitive to frequencies up to $20 \mathrm{kHz}$ and is placed $5 \mathrm{~cm}$ from the impact plate. The impact acoustic signal is sampled at $44.1 \mathrm{kHz}$.

\subsection{Collection of samples}

"Levant"-type hazelnuts collected from an orchard in Duzce, Turkey, in August 2006, are used in this experimental study. Developed hazelnuts are first selected by a standard air fan system and resorted using their measured weights. Hazelnuts less than $0.9 \mathrm{~g}$ are accepted as empty and removed from the fully developed class. The shells of fully developed hazelnuts are visually inspected and are further classified as nuts with regular shell and nuts with cracked shell. Each selected hazelnut is dropped on the metal plate and the resulting acoustic signals (Figure 3 ) are recorded. Averaged time-frequency maps of cracked and open hazelnut acoustics are given in Figures $3(\mathrm{c})$ and $3(\mathrm{~d})$.

\section{METHODS}

Before explaining the details of the proposed signal processing and classification system, let us summarize the overall algorithm. The proposed method implements an offline learning step to extract the most discriminative time-frequency features. This is achieved by first segmenting the training signals along the time axis with a pyramidal tree. In particular, the segmentation is calculated by pruning the pyramidal tree from bottom to top to maximize the KullbackLeibler distance between the expansion coefficients of good and cracked hazelnuts in each segment. The expansion coefficients in each segment are obtained from local cosine packets that provide local spectral representations. Then, each adapted time segment is decomposed into subbands by an undecimated wavelet transform. The subbands are represented in a binary tree format and are pruned to find the most discriminative subbands along the frequency axis. Finally a time-frequency map is computed by extracting the 


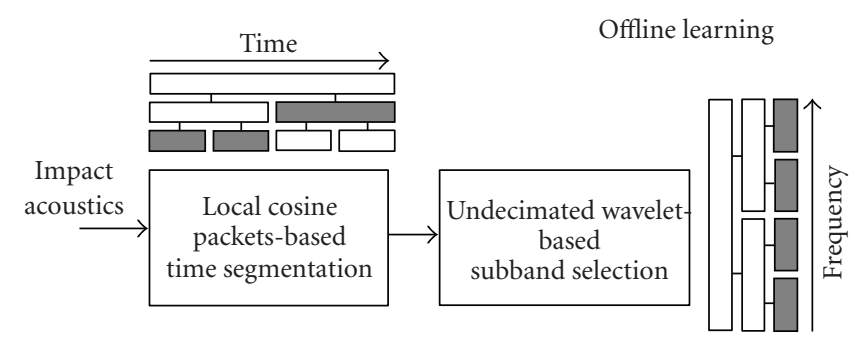

FIGURE 1: The block diagram of the offline learning step of the proposed algorithm.

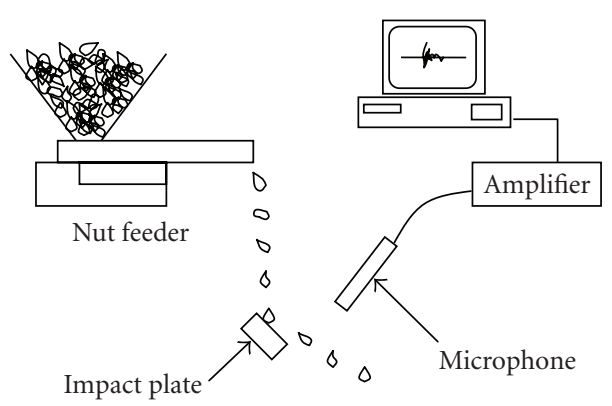

FIgURE 2: Schematic of experimental apparatus for collecting acoustic emissions from hazelnut kernel.

most relevant features. An LDA classifier is trained with these features and tested using data that was not used for training.

The main contribution of the proposed approach is the systematic and automatic extraction of the relevant features during the training step so as to improve classification accuracy. In the remainder of this section we describe that step in detail.

\subsection{Local discriminant bases}

In previous studies, impact acoustic classification is performed by combining the features obtained from the time and frequency domains as indicated in [7]. Here, we explore a different approach that is based on extracting features from the time-frequency plane. The local discriminant bases (LDBs) method was developed to extract such local information [9] for classification. The LDB algorithm basically expands the signal by using wavelet packets or local trigonometric bases over a pyramidal-binary tree as shown in Figure 1. This tree is then pruned from bottom to top to maximize a predefined cost function which measures the discrimination power of each node. The pruning operation adapts the tree for classification task. The original algorithm implements adaptation either in time or frequency. It has been shown that adaptation along both axes is crucial $[10,13]$. Once the segmentation is accomplished the timefrequency features are sorted according to a cost measure and fed to a classifier for final decision. Since the time-frequency plane is a high-dimensional space, a postprocessing step is implemented by several authors to boost the classification performance $[10,14]$. Depending on the problem, this step can be principal component analysis or a Mel-Scale-based approach to get band features.

Here, we utilize the local cosine packets and wavelet transform sequentially. As a first step to adapt to the temporal variability between the cracked and undamaged hazelnut acoustics, we use local cosine packets which provide time axis segmentation with smooth windows. Local cosine packets are widely used in signal processing to segment signals with time varying characteristic [15]. Once we obtain the time axis segmentation, we use wavelet transform to select the most relevant subbands for the final feature extraction. Since our purpose is to discriminate between signals coming from different classes, we use a dissimilarity criterion to obtain the segmentations along both the time and frequency axis. Now let us describe the distance measure and algorithms used for time and frequency segmentation in detail.

\subsection{Dissimilarity measure}

Various types of dissimilarity measures were tested and the following ones were selected and used. Let $p$ and $q$ be the spectral energy distributions of signals belonging to class 1 and class 2 , respectively. The distance measure can be:

(i) the symmetric Kullback-Leibler distance, which is also called $J$-divergence:

$$
\begin{aligned}
& J(p, q)=I(p, q)+I(q, p), \\
& I(p, q)=\sum_{i=1}^{n} p_{i} \log \frac{p_{i}}{q_{i}},
\end{aligned}
$$

or

(ii) Euclidean distance:

$$
D(p, q)=\|p-q\|^{2}=\sum_{i=1}^{n}\left(p_{i}-q_{i}\right)^{2} .
$$

We have used the $J$ criterion for time segmentation and $D$ for subband selection in each adapted segment. As shown in Figure 4(a), the averaged spectrum of cracked and regular hazelnut shells has most of its energy in midbands. However, when the distance between these two spectra is calculated, we noticed that the $J$ criterion emphasizes higher bands more than the $D$ criterion. During our experimental studies, we observed that the most discriminant locations are located in higher frequency bands. Therefore, using $J$ for time segmentation provided better results. 


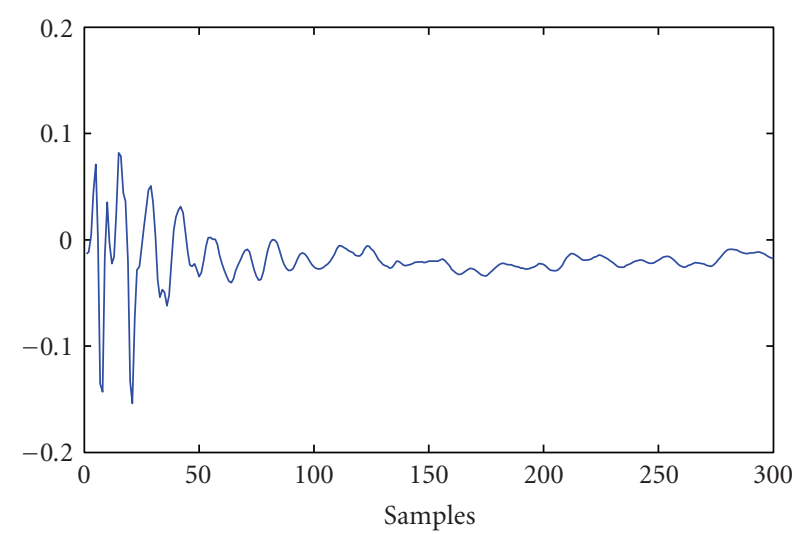

- ReH

(a)

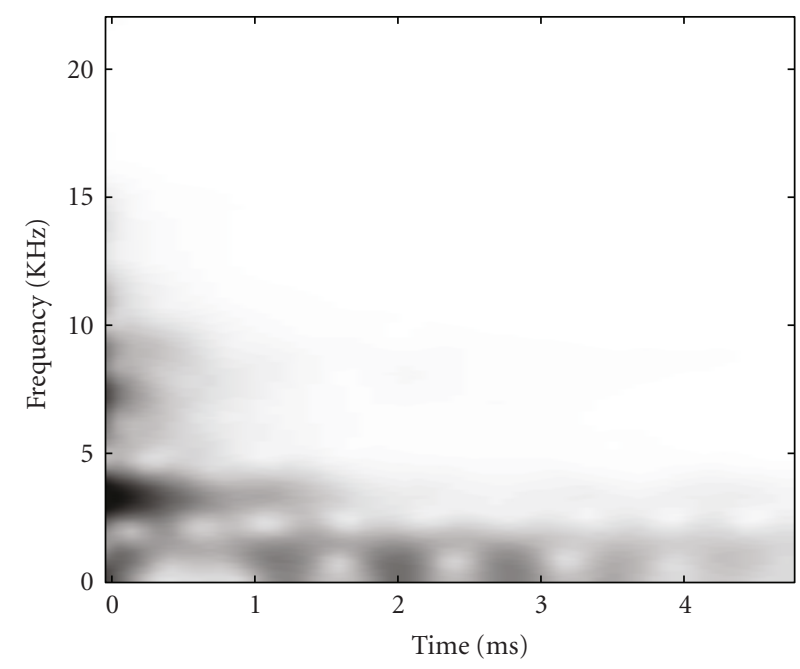

(c)

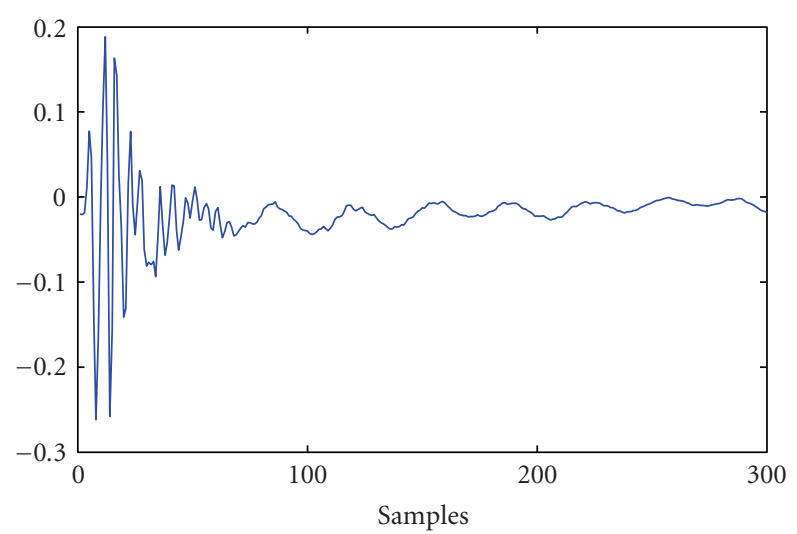

$-\mathrm{CrH}$

(b)

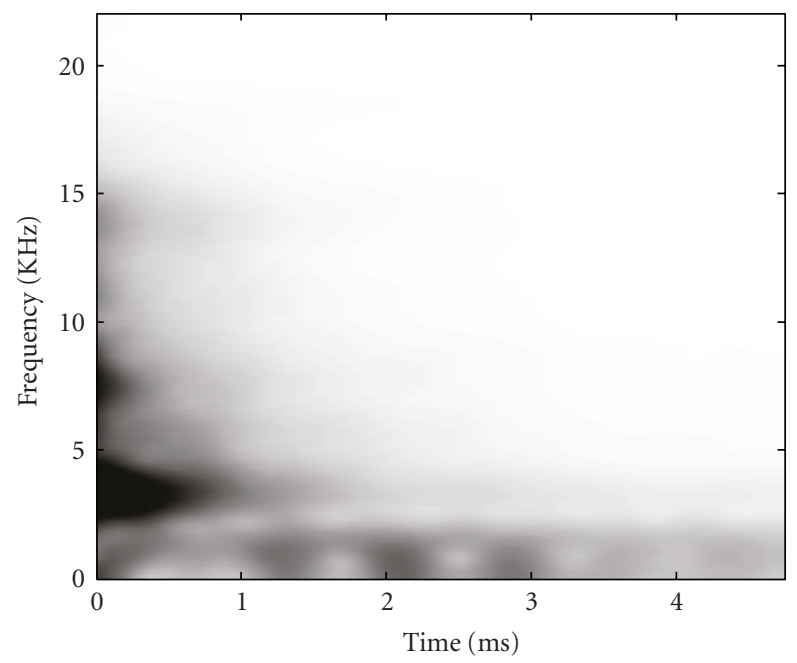

(d)

FIGURE 3: Typical impact acoustic signals of (a) fully developed hazelnuts with regular shell (ReH), (b) fully developed hazelnuts with cracked shell $(\mathrm{CrH})$, and averaged spectrogram of (c) $\mathrm{ReH}$ and (d) $\mathrm{CrH}$ signals.

\subsection{Time segmentation with local cosine packets}

The impact acoustic signals have different characteristics in the impact, postimpact, and late impact phases. Therefore, impact signals should be analyzed locally. In general, local information of the signal is extracted by a short time Fourier transform (STFT). Some researchers used local cosine packets (LCPs) because of its advantages over the STFT $[9,11]$. Local cosine packets (LCPs) is preferred in this study and used to partition the time axis in a pyramidal tree structure of Figure 1.

Local cosine packets partition the time axis by using smooth bells [15] that are constructed using cut-off functions $r(t)$ that satisfy

$$
\begin{gathered}
\left|r\left(t^{2}\right)\right|+\left|r(-t)^{2}\right|=1 \quad \forall t \in R, \\
r(t)= \begin{cases}0 & \text { if } t \leq-1, \\
1 & \text { if } t \geq 1 .\end{cases}
\end{gathered}
$$

An example of such a function $r(t)$ is

$$
r(t)= \begin{cases}0 & \text { if } t \leq-1, \\ \sin \left[\frac{\pi}{4}\left(1+\sin \left(\frac{\pi t}{4}\right)\right)\right] & \text { if }-1<t<1, \\ 1 & \text { if } t \geq-1 .\end{cases}
$$

First, all signals are represented with local cosine packets within smooth windows (as in (4) in the tree structure. The resulting expansion coefficients are squared and then averaged over the signals in the given class. This provides an averaged energy spectrum of each class in a given time segment within the pyramidal tree. Let $p_{i}$ and $q_{i}$ be the mean energy spectra of cracked and regular classes, in a given time segment, respectively. The distance between the average spectra is calculated with the criterion $J$ where " $n$ " in (1) corresponds to the total number of time samples in a given node. This way, the distance is accumulated along the spectrum within 


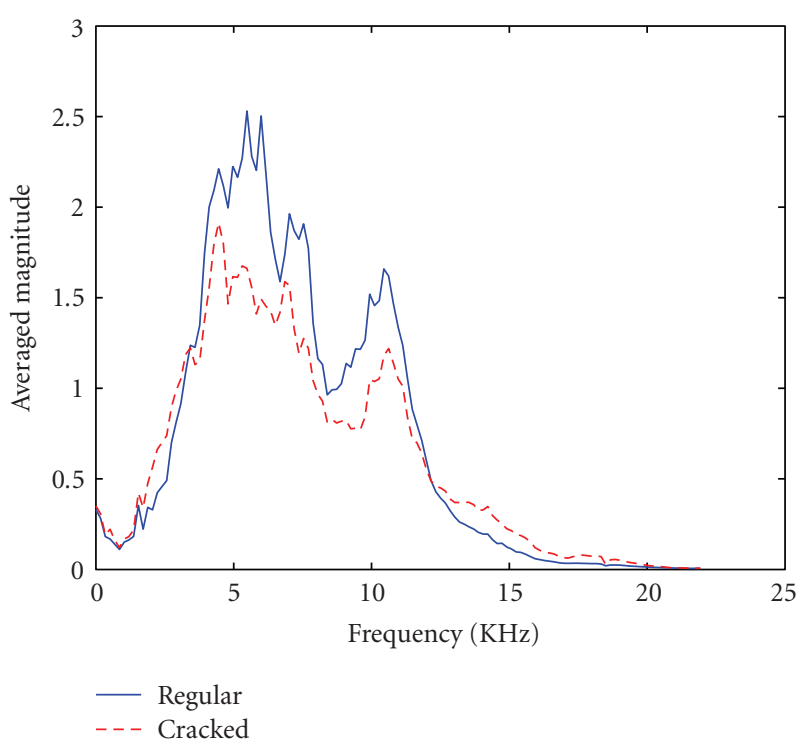

(a)

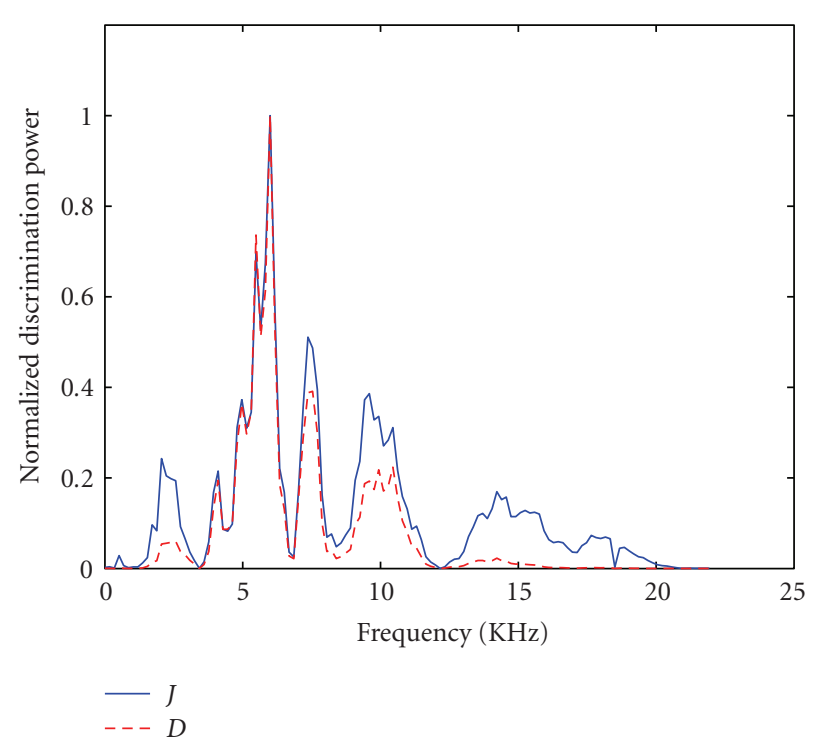

(b)

FIGURE 4: (a) The averaged magnitude spectrum of cracked and regular hazelnut impact acoustic signals related to the first 128 samples.

(b) The J and D distance between two spectra.

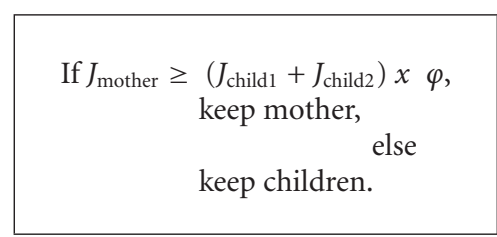

Algorithm 1: Pruning algorithm.

all subspaces to get a single value representing each node of the tree. The resulting binary tree is then pruned from bottom to top according to the rule in Algorithm 1 to find the nodes with maximum discrimination power:

Here $J_{\text {mother }}$ and $J_{\text {child }}$ are the discrimination power of the mother and children nodes and are computed by the Kullback-Leibler distance criteria and $\varphi$ is an empirically selected constant. It is experimentally found that $\varphi=0.95$ preserves discriminative information while leading to robust segmentation. The algorithm keeps the mother if it captures $95 \%$ of the discriminative power of the children, otherwise it keeps the children.

\subsection{Frequency segmentation}

We have observed time jitter in the recorded signals which is due to variances in the travel time to the steel plate. Therefore, a shift invariant decomposition is highly desirable for processing the signal. The importance of shift invariance for classification is also emphasized in [9-11]. The undecimated wavelet transform (UDWT) has the shift-invariance property. It was first used for texture classification in [16]. In this study, a similar approach is taken to analyze the impact sig- nals for classification. A filter $f(n)$ with a $z$-transform $F(z)$ that satisfies the quadrature mirror filter condition

$$
F(z) F\left(z^{-1}\right)+F(-z) F\left(-z^{-1}\right)=1
$$

is used to construct the pyramidal filter bank (Figure 5). The high-pass filter $g(n)$ is obtained by shifting and modulating $f(n)$. Specifically, the $z$ transform of $g(n)$ is chosen as

$$
G(z)=z F\left(-z^{-1}\right) .
$$

The subsequent filters in the filter bank are then generated by increasing the width of $f(n)$ and $g(n)$ at every step, for example,

$$
\begin{aligned}
& F_{i+1}(z)=F\left(z^{2^{i}}\right), \\
& G_{i+1}(z)=G\left(z^{2^{i}}\right), \quad i=0,1, \ldots, N .
\end{aligned}
$$

In the signal domain, the filter generation can be expressed as

$$
\begin{aligned}
& f_{i+1}(k)=[f]_{12^{2}}, \\
& g_{i+1}(k)=[g]_{12^{i}},
\end{aligned}
$$

where the notation []$_{1 m}$ denotes the up-sampling operation by a factor of $m$.

The resulting filter bank of which the second level frequency response is demonstrated at Figure 6 is used to extract the subband signals at the nodes. It is observed that the signal has different energy distribution in each subband.

The Euclidean distance between cumulative probability distributions (cdf) of subband energies in (2) is chosen as the discriminative measure. We selected to use cdf over pdf because it is easier to calculate. One can also use pdf instead. The resulting pyramidal subband tree is pruned from bottom to top by the rule, shown in Algorithm 2. 


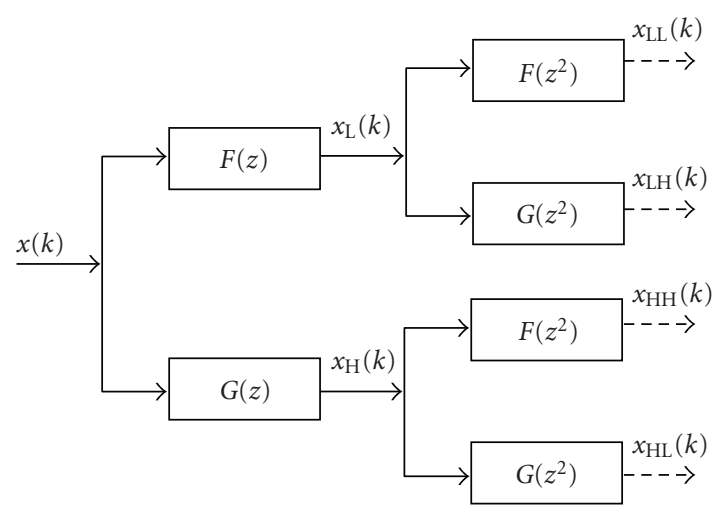

Figure 5: Pyramidal filter tree up to second level. L and $\mathrm{H}$ stand for low and high bands, respectively.

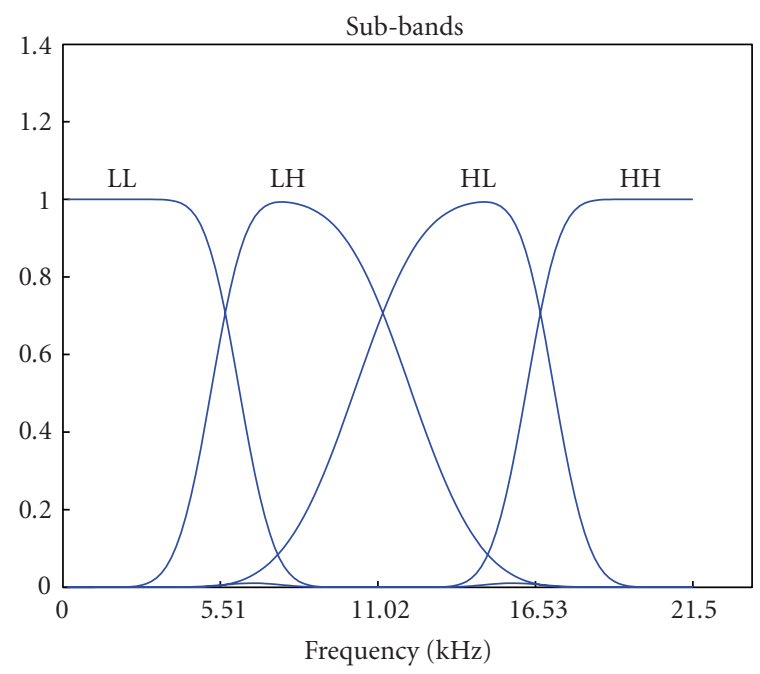

Figure 6: Frequency response of the 2nd level filters.

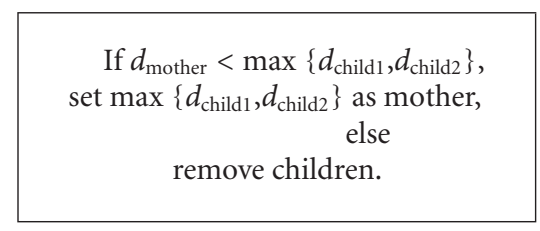

Algorithm 2: Pruning algorithm.

Where $d_{\text {child } 1}$ and $d_{\text {child } 2}$ are the Euclidian distances of subbands nodes of mother node where as $d_{\text {mother }}$ is the distance of the mother node.

\section{RESULTS}

One thousand cracked and one thousand uncracked hazelnut kernels are used in this study. Each hazelnut is dropped on the metal plate and the resulting acoustic signal consisting of 768 time samples is recorded. We analyzed the signal up to a tree depth of 4 resulting in a smallest segment size of 48 time samples in the time domain. We empirically found that this level provides a healthy balance between focus on to transient waveforms and the required spectral resolution to distinguish between subbands with different behavior. The signals were first represented by using LCP over the pyramidal tree structure. The pyramidal tree was pruned by using the algorithm of Section 3.3 and the adaptive time segmentation for classification purpose was obtained for different sets of signals as indicated in Figure 7. It was observed that different sets of signals may cause different segmentation in time. We used the segmentation of Figure 7(a) in our simulations. In this case, the time axis is divided into 7 segments.

In each time segment, the signal was decomposed into subbands up to the 4 th wavelet decomposition level and the most relevant subbands were detected by using the procedures of Section 3.4.

A discriminative time-frequency map was generated in Figure 8 by combining the adaptively pruned trees both in time and frequency to visualize the most crucial $t-f$ patterns. In our application, the algorithm usually generates a $t$ - $f$ map with around 70 subbands for various training data sets. For every signal in each training set, the energy value for each subband was computed resulting in two sets of feature vectors corresponding to cracked and healthy shell classes.

The 70 features obtained were sorted in descending order according to their discrimination power and then used for classification. Fisher's discrimination measure is used for feature selection. We observed with all training data sets that the most discriminative feature locations were concentrated in the high frequency bands corresponding to the early and post impact regions as indicated in Figure 8. Among the 70 subbands, the 25 most discriminative ones are indicated by different shades of gray, with darker shades corresponding to higher discrimination levels.

\subsection{Classification}

In order to assess the efficiency of the proposed algorithm, a comparison is made with the features of [7] and those features of our previous work [8] which used nonadaptive subbands and different order statistical features. Recall that in [7], 70 features were extracted from the short time variances of signal; maximum signal amplitude, spectral peak locations, and Weibull distribution fit to the envelope of the impact signal and all are used for classification. In the subbandbased algorithm [8], features were extracted from subband signals and the 20 most relevant features and the subbands including these features were manually selected. The time segmentation of Figure 7(a) is employed to obtain a total of 28 statistical features including mean absolute energy, variance, skewness, and kurtosis on each of the seven time segments.

The one thousand acoustic signals for each class are randomly divided into 5 nonoverlapping sets, each consisting of 200 records. Five pairs of uncracked and cracked sets are then randomly formed. Each pair is used to construct the adaptive $t-f$ segmentation and select features. The features identified are then used with the remaining 1600 acoustic signals to determine the performance of the classifier. This procedure is repeated five times with the five different pairs of uncracked and cracked sets. 


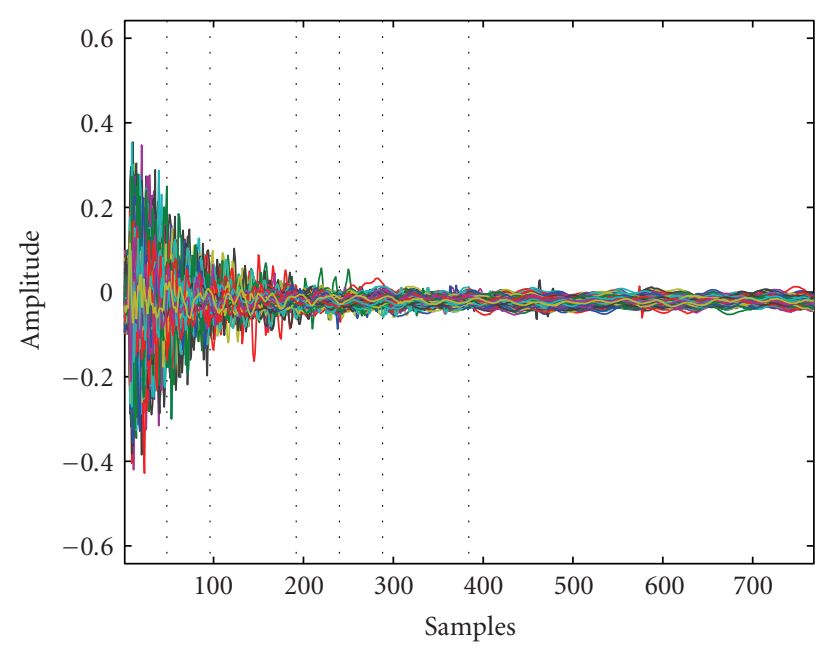

(a)

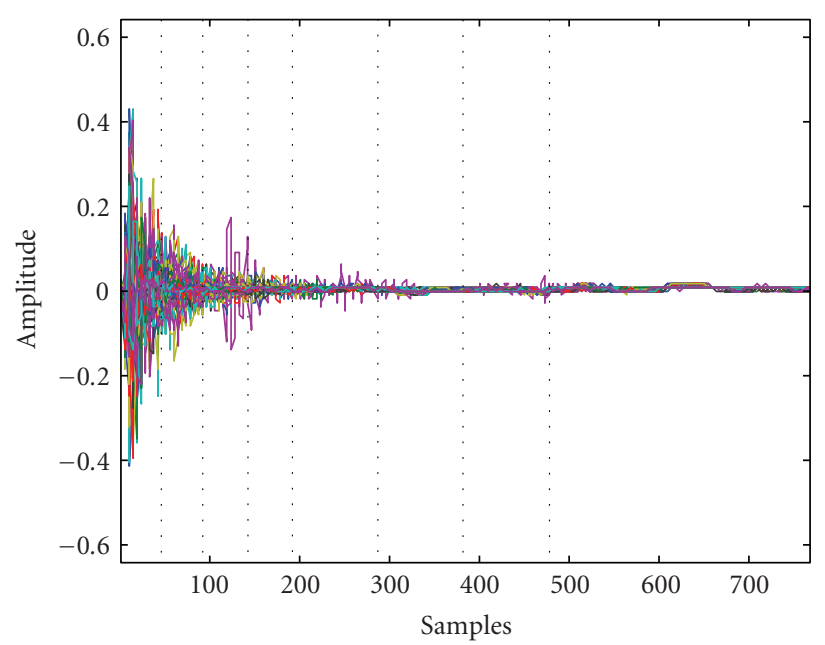

(b)

Figure 7: The adaptive time segmentation grids (dotted lines) of (a) set1 and (b) set2.

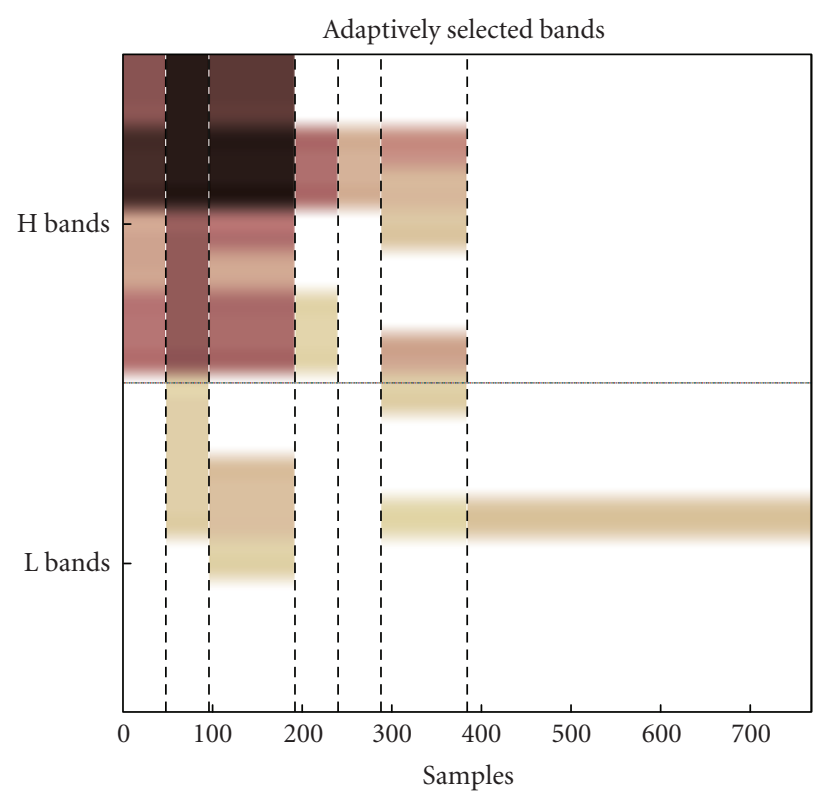

FIGURE 8: The time-frequency discrimination map of impact acoustic data. Darker regions indicate higher discrimination power.

The optimal number of features for classification was investigated by adding features one by one according to Fisher's discrimination criterion. This step is repeated for all four methods. Related classification error curves are presented in Figure 9.

We noticed that the lowest classification error is achieved with our proposed approach. The minimal classification error rates achieved by each method are given in Table 1. It is observed that the lowest error is achieved by the first 64 features with an error level of $3.5 \%$ by our proposed approach. For the method of [7], 43 out of 70 time and frequency domain features provided the minimum er-

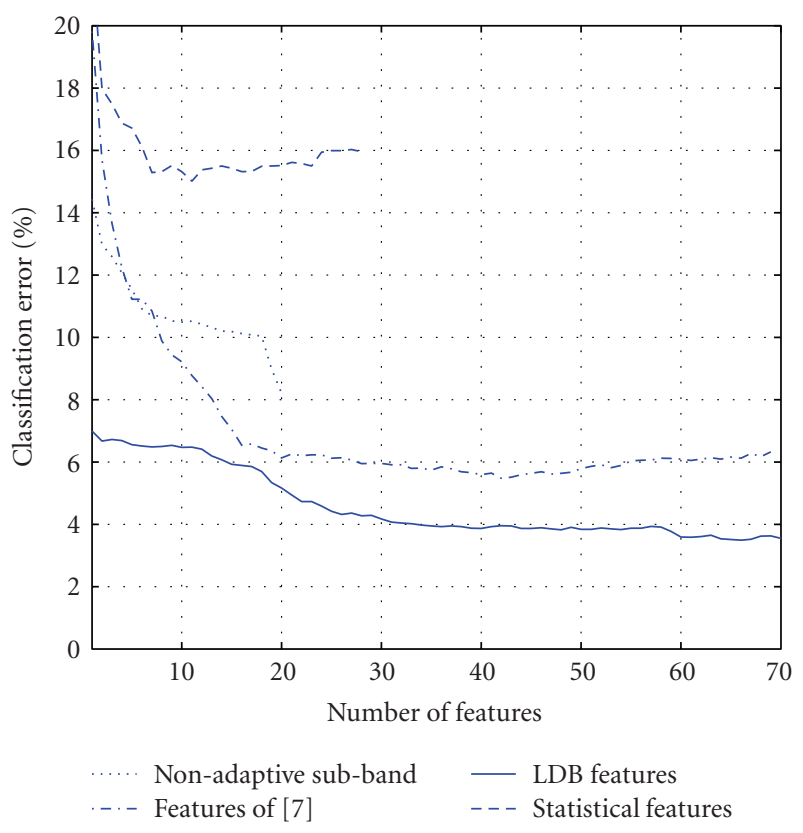

FIgURE 9: The classification error rates with various numbers of features.

ror level. Similarly, 20 nonadaptive subband features are used for the method of [8]. The statistical features gave poor classification error rates compared to other methods. The lowest error rate occurred when the first 7 features are used. Our proposed approach reaches an error rate around $4 \%$ after the first 30 features. Increasing the number of features provided marginal improvement of the error rate.

The ROC curves for the three methods are presented in Figure 10. It is observed that 64- and 30-dimensional LDB features provide higher detection of cracked hazelnuts for a given false alarm rate. 
TABLE 1: Classification rate comparison of proposed LDB-based method against the previously developed algorithms.

\begin{tabular}{lc}
\hline Method & Accuracy $(\%)$ \\
\hline 43 features of method of [7] & 94.47 \\
7 statistical features & 85.00 \\
20 nonadaptive subband features & 91.80 \\
64 LDB-based feature & $\mathbf{9 6 . 5 1}$ \\
\hline
\end{tabular}

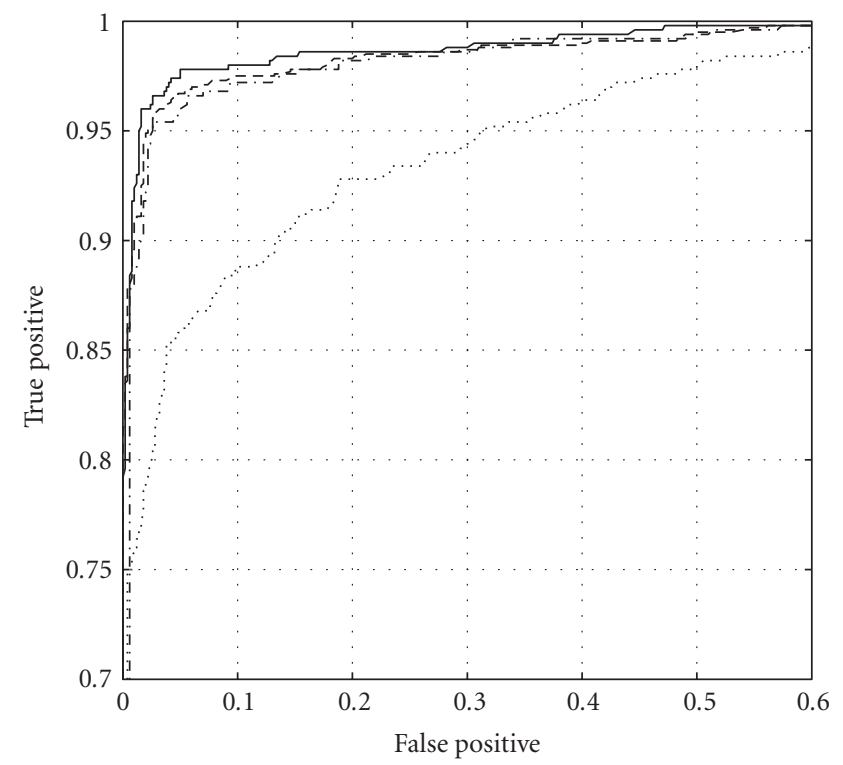

$$
\begin{array}{ll}
\text {.... } 20 \text { non-adaptive sub-band } & -64 \text { LDB features } \\
\text {..- } 43 \text { features of [7] } & --30 \text { LDB features }
\end{array}
$$

FIgURE 10: Receiver operating characteristics (ROCs) curves.

\subsection{Filter selection}

Various types of wavelet filters (Daubechies, Coiflet, and Sym) are used for decomposition of the frequency axis, and their effects on classification accuracy are observed. In Figures 11(a) and 11(b), the classification accuracy of Daubechies and Coiflet wavelets is depicted in contour graphics format. The $x$-axis indicates the total number of features retained after sorting. The $y$-axis indicates the filter type used in subband decomposition. The higher filter types correspond to higher-order filters. The darker regions in the contour graph give lower classification accuracy. It is observed that better classification error rates $(<4 \%)$ are obtained when approximately 40 or more features are retained after decomposition with high-order wavelet filters (Db12Db15 and Coif3-Coif5). We selected one of the high-order wavelet filters, Coiflet 4, for further analysis. The discriminant band distribution of Figure 8 may slightly change depending on the wavelet filter.

\subsection{Effect of noise on classification}

In order to asses the robustness of our methods against disturbing effects, a zero mean Gaussian noise at various SNR levels is added to the signal, and classification performances are compared as shown in Figure 12. It is observed that the algorithm performs well for reasonable noise levels. The algorithm usually selects low level subbands nodes when the signals are disturbed by high-level noise. This can be justified by fact that the energy of the impact acoustics is concentrated in the mid and lower bands of the spectrum as indicated in Figure 4. In order to keep the efficiency in classification, the algorithm selects features from lower bands with increasing noise level. This also results a decrease in classification accuracy.

\subsection{Effect of shift-invariance to classification}

As indicated in the previous sections the main motivation for using UDWT against DWT is the shift invariance property of the UDWT In order to justify our selection we compared the UDWT results with those obtained from the DWT and spincycle procedure of [17]. The spin-cycle procedure is introduced by [17] to overcome the lack of shift invariance of the DWT and LCP. In particular, a signal is shifted to the left and right for a selected number of spins. For each shift, the signal is expanded into its DWT coefficients. These coefficients are either averaged or processed individually. It has been shown that the spin-cycle procedure provides many improvements over the direct use of the DWT or LCP $[13,17]$. In Figure 13, we show the classification curves obtained from the DWT, the DWT with spin-cycle, and the UDWT methods.

As expected, the results obtained from DWT were poor. Interestingly the DWT with spin-cycle provided results as good as the UDWT. We note that the minimum error of spincycle method was slightly lower than UDWT but used more features. However, one should note that the computational complexity of spin-cycle method is 3 times higher than that of UDWT. In real-time applications, it is difficult to obtain fast processing by this method.

\subsection{Feature selection}

A total of 210 features corresponding to 210 time-frequency band are obtained before frequency axis pruning operation. Recall that when Fisher criterion is used for feature sorting, the frequency tree is pruned as a prior step to obtain an uncorrelated subband feature set. Here, we investigate the efficiency of the proposed approach by comparing it to the correlation-based feature selection (CSF) procedure of [12]. The CSF uses the feature-to-class and feature-to-feature correlations to select a subset of features from a redundant set. Since it can account for the feature-to-feature correlations, we presented the unpruned full feature dictionary to CSF method. The subset returned by the CSF method was used for classification. In Figure 14, we show the classification curve of CSF and compare it with the curve of our algorithm based on Fisher's criterion on the pruned set. The CSF method achieved to minimal error of $4 \%$ with around 70 features. Although a redundant feature dictionary was presented to the algorithm, it successfully selected a subset without any pruning step. 


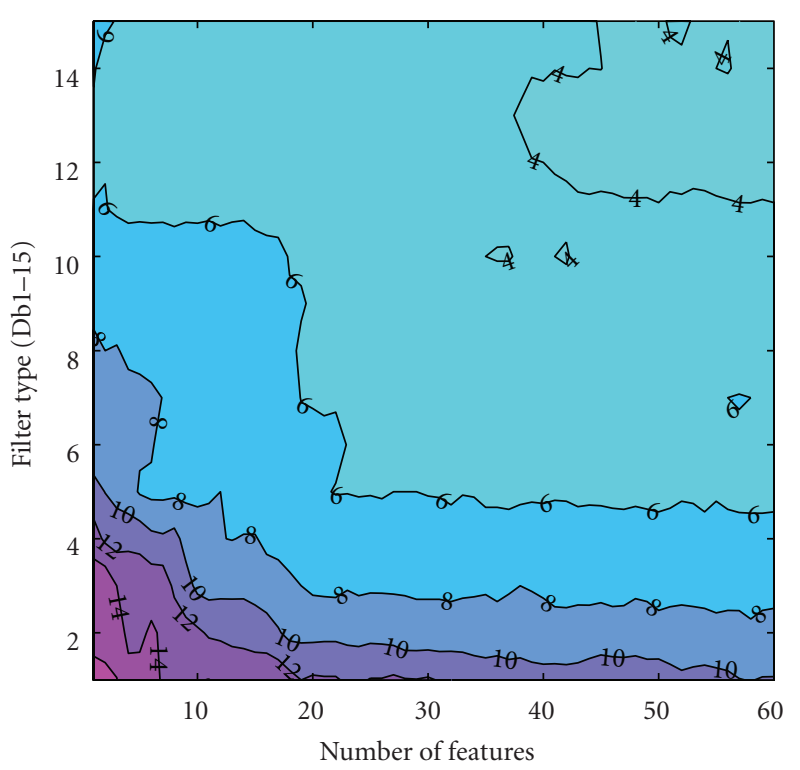

(a)

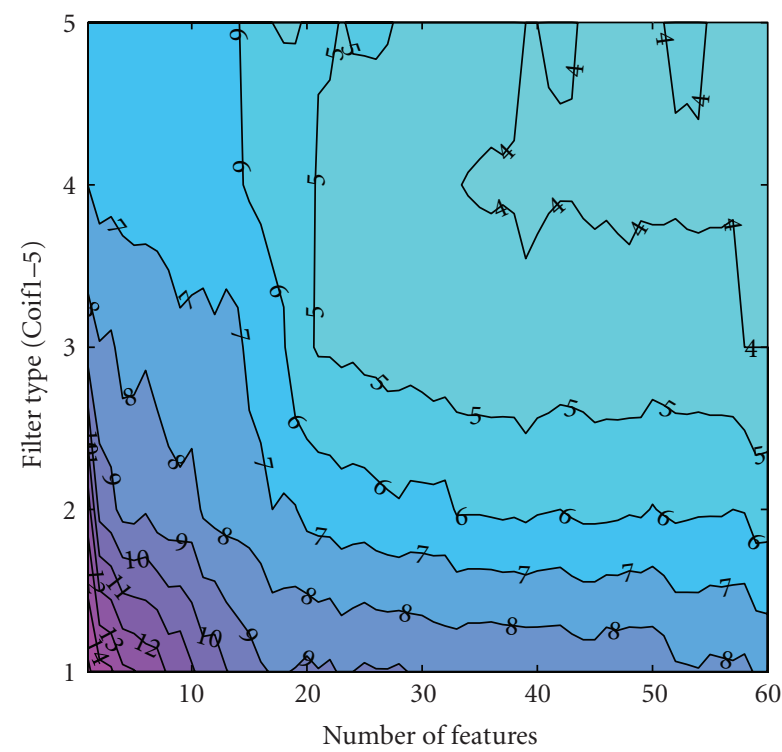

(b)

FIGURE 11: The effect of selected wavelets and feature dimension on classification accuracy; (a) Daubechies, (b) Coiflet.

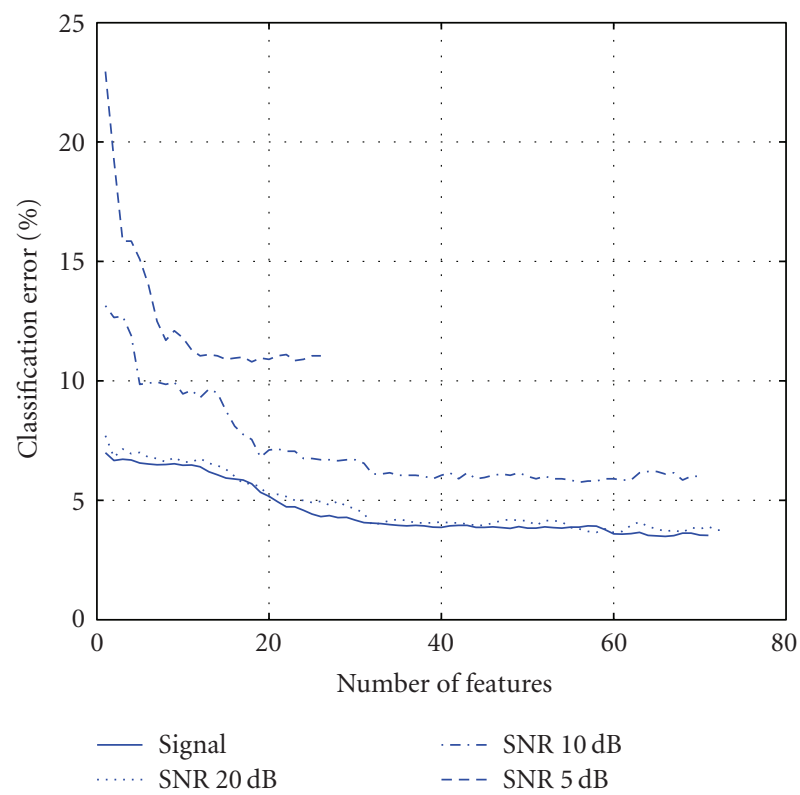

FIgURE 12: The classification error curves for noise disturbed impact acoustic signals.

It is observed that the classification error increased after 70 features. Interestingly within the first 10 features, the CSF provides a lower error rate than Fisher's criterion. However, with increasing number of features the Fisher-based sorting procedure over the pruned subband tree provided lower error rates. The pruning algorithm in our method automatically eliminated two third of these features. The error curve (Pruned tree, Fisher) in Figure 14 indicates that the pruning and Fisher criteria combination is successful at detecting relevant features in acoustic signals.

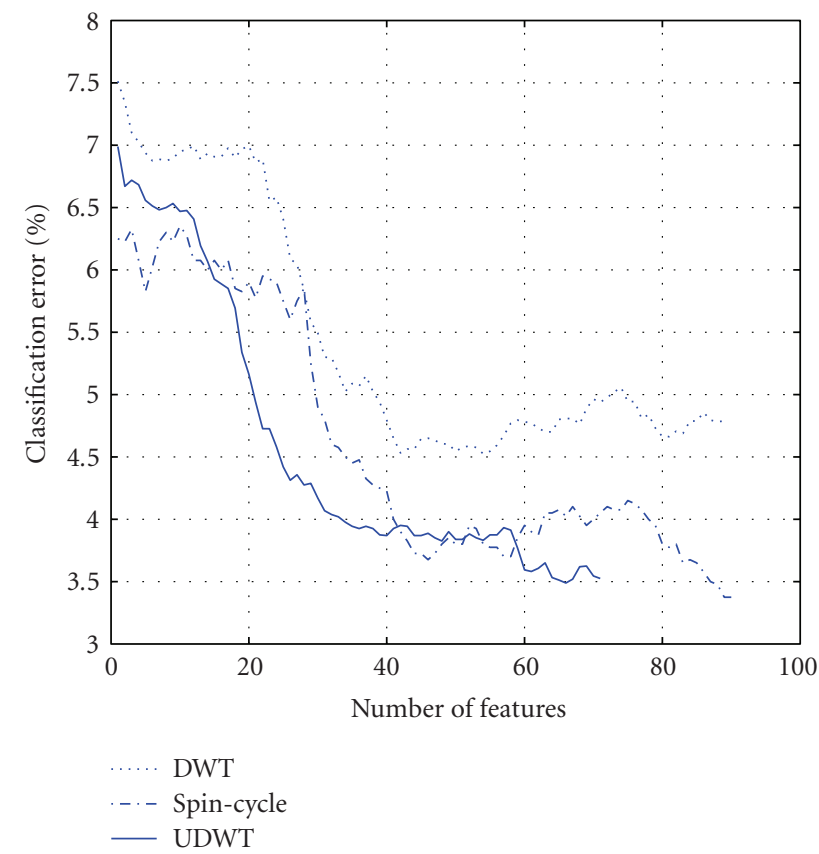

Figure 13: The classification error curves for evaluating the efficiency of shift invariance property. The spin-cycle curve stands for the results obtained from DWT supported 1-Spin-cycle procedure.

\subsection{Computational complexity}

Determining the best time-frequency segmentation of the signals and the bands to be retained for classification is relatively computationally demanding but this step has to be carried out only once, offline. For online processing, the throughput of the algorithm in terms of nuts processed per second depends on the number of features used in 


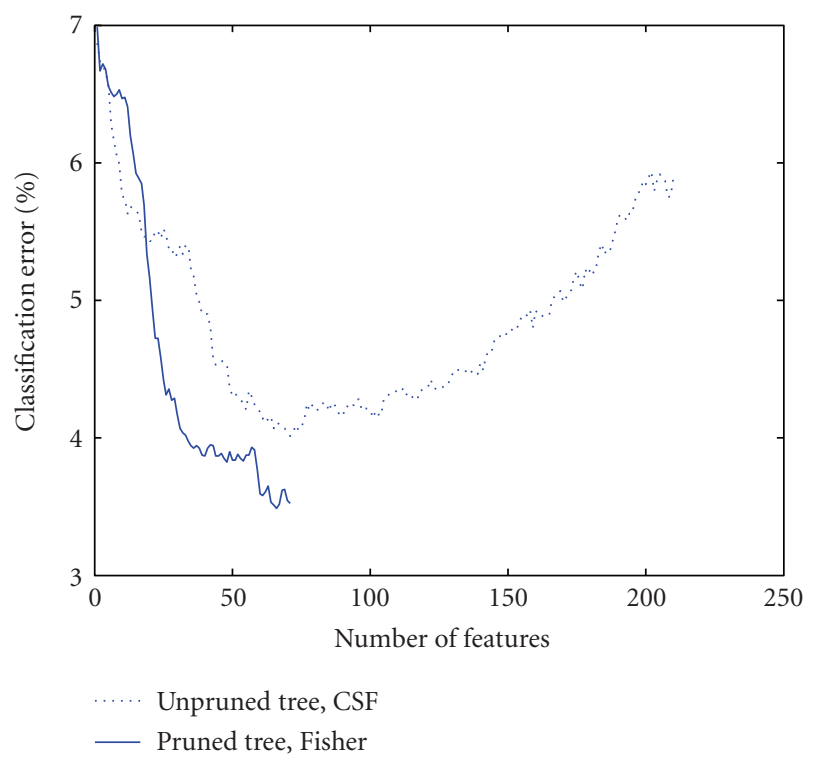

FIGURE 14: The classification error curves of CSF method and our proposed approach.

classification. When the first 64 features providing the best classification rate is employed, all 768 samples need to be processed. In this case 17.4 milliseconds are required for signal acquisition of a single nut at a sampling rate of $44.1 \mathrm{kHz}$. The computations for feature extraction and classification require 13.1 milliseconds on a dedicated $\mathrm{P} 43 \mathrm{GHz}$ processor. In this case, up to 32 nuts can be processed in a second with classification error of $3.5 \%$. In case an extra $0.5 \%$ classification error is tolerable, up to 45 nuts can be processed in a second with 30 features. We observed that only the first half of the signal is required to compute the first 19 features. The classification error achievable at this case is $5.3 \%$ and the throughput can be as high as 119 nuts/s provided that the mechanical sorter system is able to keep up with signal processing.

\section{CONCLUSION}

In this study, an adaptive time frequency plane feature selection algorithm is introduced to separate cracked hazelnuts from regular hazelnuts. The adaptation in time and frequency is achieved by combining local cosine packets and an undecimated wavelet transform. The impact signal is adaptively segmented in the time domain with LCP. Similarly the signals in each resulting time segment are decomposed into subbands by an undecimated wavelet transform. The subband tree is pruned from bottom to top according to the discrimination power of its nodes. The resulting $t-f$ map is used to extract the best features for classification. Interestingly, higher bands are selected by the algorithm. Finally, the hazelnuts are classified by LDA. The proposed approach is robust, adaptive to signal type and provides superior classification results. The algorithm can work in a real time automatic sorter with a processing speed of 45 nuts/s.

\section{ACKNOWLEDGMENTS}

This work is supported by National Science Foundation (NSF) and by the Project EEEAG-106E057 and Program 2214 of National Scientific Research Council of Turkey.

\section{REFERENCES}

[1] C. R. Dichter, "Risk estimates of liver cancer due to aflatoxin exposure from peanuts and peanut products," Food and Chemical Toxicology, vol. 22, no. 6, pp. 431-437, 1984.

[2] T. Pearson, "Separating early split from normal pistachio nuts for removal of nuts contamination on the tree with aflatoxin," M.S. thesis, University of California, Berkeley, Calif, USA, 1987.

[3] T. Pearson, "Machine vision system for automated detection of stained pistachio nuts," Lebensmittel-Wissenschaft undTechnologie, vol. 29, no. 3, pp. 203-209, 1996.

[4] T. Pearson and T. F. Schatzki, "Machine vision system for automated detection of aflatoxin-contaminated pistachios," Journal of Agricultural and Food Chemistry, vol. 46, no. 6, pp. 22482252, 1998.

[5] T. Pearson, "Detection of pistachio nuts with closed shells using impact acoustics," Applied Engineering in Agriculture, vol. 17, no. 2, pp. 249-253, 2001.

[6] A. E. Cetin, T. Pearson, and A. H. Tewfik, "Classification of closed- and open-shell pistachio nuts using voice-recognition technology," Transactions of the American Society of Agricultural Engineers, vol. 47, no. 2, pp. 659-664, 2004.

[7] I. Onaran, B. Dulek, T. Pearson, Y. Yardimci, and E. Çetin, "Detection of empty hazelnuts from fully developed nuts by impact acoustics," in Proceedings of the 13th European Signal Processing Conference (EUSIPCO '05), Antalya, Turkey, September 2005.

[8] H. Kalkan and Y. Yardimci, "Classification of hazelnuts by impact acoustics," in Proceedings of the 16th IEEE Signal Processing Society Workshop on Machine Learning for Signal Processing (MLSP '06), pp. 325-330, Maynooth, Ireland, September 2006.

[9] N. Saito and R. R. Coifman, "Local discriminant bases," in Wavelet Applications in Signal and Image Processing II, vol. 2303 of Proceedings of SPIE, pp. 2-14, San Diego, Calif, USA, July 1994.

[10] N. F. Ince, A. H. Tewfik, and S. Arica, "Classification of movement EEG with local discriminant bases," in Proceedings of IEEE International Conference on Acoustics, Speech and Signal Processing (ICASSP '05), vol. 5, pp. 413-416, Philadelphia, Pa, USA, March 2005.

[11] N. F. Ince, A. H. Tewfik, and S. Arica, "Extraction subjectspecific motor imagery time-frequency patterns for single trial EEG classification," Computers in Biology and Medicine, vol. 37, no. 4, pp. 499-508, 2007.

[12] M. A. Hall, "Correlation-based feature selection for machine learning," Ph.D. dissertation, Department of Computer Science, Waikato University, Hamilton, New Zealand, 1998.

[13] N. F. Ince, S. Arica, and A. H. Tewfik, "Classification of single trial motor imagery EEG recordings with subject adapted non-dyadic arbitrary time-frequency tilings," Journal of Neural Engineering, vol. 3, no. 3, pp. 235-244, 2006.

[14] K. Englehart, B. Hudgins, P. A. Parker, and M. Stevenson, "Classification of the myoelectric signal using timefrequency based representations," Medical Engineering and Physics, vol. 21, no. 6-7, pp. 431-438, 1999. 
[15] M. V. Wickerhauser, Adapted Wavelet Analysis from Theory to Software, A. K. Peters, Natick, Mass, USA, 1994.

[16] M. Unser, "Texture classification and segmentation using wavelet frames," IEEE Transactions on Image Processing, vol. 4, no. 11, pp. 1549-1560, 1995.

[17] N. Saito, R. R. Coifman, F. B. Geshwind, and F. Warner, "Discriminant feature extraction using empirical probability density estimation and a local basis library," Pattern Recognition, vol. 35, no. 12, pp. 2841-2852, 2002. 